\title{
Pseudospin valve in bilayer graphene: towards graphene-based pseudospintronics
}

\author{
P. San-Jose, E. Prada, E. McCann, and H. Schomerus \\ Department of Physics, Lancaster University, Lancaster, LA1 4YB, United Kingdom
}

(Dated: June 18, 2009)

\begin{abstract}
We propose a non-magnetic, pseudospin-based version of a spin valve, in which the pseudospin polarization in neighboring regions of a graphene bilayer is controlled by external gates. Numerical calculations demonstrate a large on-off ratio of such a device. This finding holds promise for the realization of pseudospintronics: a form of electronics based upon the manipulation of pseudospin analogous to the control of physical spin in spintronics applications.
\end{abstract}

PACS numbers: 75.70.Ak, 75.47.Pq, 85.75.-d

Soon after its discovery [1, it was realized that graphene supports an additional quantum number called pseudospin [2, 3, 4, that arises because the honeycomb lattice is composed of two triangular sublattices. Wavefunction amplitudes can be written like the two components of a spin- $1 / 2$ elementary particle, and electrons in graphene display characteristics analogous to relativistic fermions [2, 3. In particular, this includes the celebrated effect of chirality, whose profound consequences include an unusual sequencing of plateaus in measurements of the quantum Hall effect [2, 3, 5], suppression of backscattering [6, 7] and Klein tunneling at interfaces [8, 9].

So far, it has not been possible to exploit the pseudospin degree of freedom in graphene in a similar way as physical spin in spintronics [10] and quantum computing [11] applications. In a monolayer of graphene, chirality means that the orientation of an electron's pseudospin is inextricably linked to the direction of its momentum, thus constraining the pseudospin to lie in the plane of the graphene sheet and preventing its use as an independently-tunable degree of freedom. In bilayers of graphene [5, 12, 13, the pseudospin degree of freedom is associated with the electronic density on the two layers. The constraint of chirality entails that electronic density is equally divided between the two layers so that the pseudospin again lies in the plane of the layers but now turning twice as quickly as the direction of momentum [5, 12. Min et al 14] were the first to realize that bilayers still offer a promising platform for pseudospintronics. In particular, they predicted that a pseudomagnetic state can form spontaneously due to strong Coulomb interactions at vanishing charge-carrier density; this effect is intimately tied to the fact that the density of states remains finite because the dispersion relation is parabolic, in contrast to the situation in a monolayer.

In this Letter we propose a variant of graphene-based pseudospintronics which exploits another direct advantage of a bilayer over a monolayer, namely the facility to induce a difference between the on-site energies on the two layers via a perpendicularly applied electric field, which can be realized by pairs of gate electrodes (see Fig. 11). The resulting asymmetry of the layers induces an energy gap between the conduction and valence
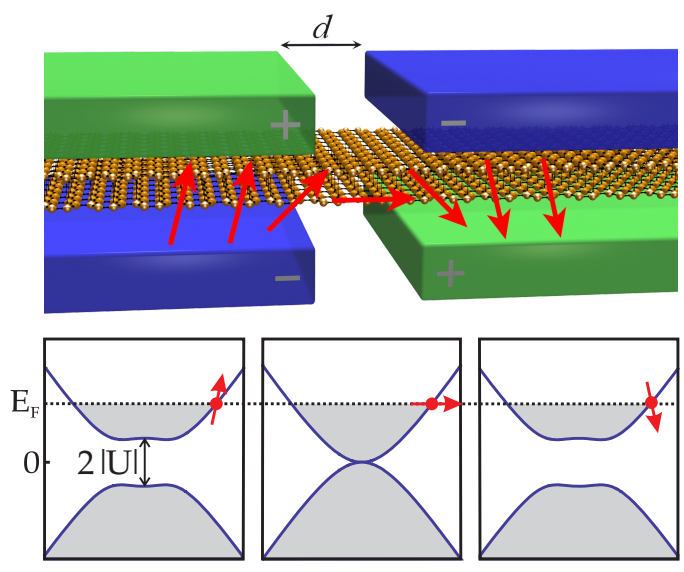

FIG. 1: Pseudospin-valve effect in bilayer graphene. Schematic diagram of a pseudospin valve in bilayer graphene in its antiparallel (AP) configuration.

bands [12, 15, 16, 17, as observed in photoemission [13] and transport [18, 19] measurements. For states above or below the gap, interlayer asymmetry has the effect of creating an "up" or "down" component of pseudospin perpendicular to the electronic momentum and the plane of the sheet [12, 14]. The electric field hence acts on the pseudospin in the same way as a magnetic field acts on the physical spin of electrons in spintronic applications. In particular, the preferred pseudospin direction can be switched by inverting the sign of the applied potential difference. In analogy to the giant magnetoresistance (GMR) induced by a domain wall boundary in magnetic materials [20, 21], one would therefore expect that interfaces between regions of different gate polarity inhibit the flow of electrons. We will demonstrate that this effect can indeed be utilized to realize an all-electronic, pseudospin-based analogue of a spin valve with a large on-off ratio.

Concepts. - The proposed pseudospin valve can be realized in a $2 D$ sheet of bilayer graphene with sets of gates which produce a spatial variation of interlayer asymmetry in the direction of current flow (see Fig. 1). Top and bottom gates are used to independently control the Fermi 


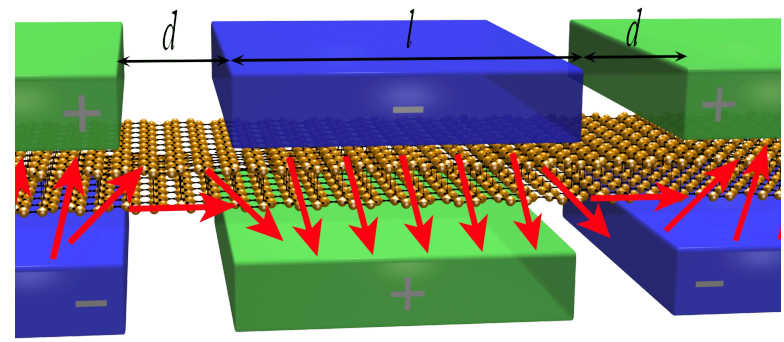

FIG. 2: Schematical illustration of a pseudospin-valve transistor. This device is operated by switching the polarity of a central gate of length $l$ (shown is the antiparallel configuration).

level and the interlayer asymmetry, the latter creating an out-of-plane component of pseudospin. When the polarity of the two pairs of gates is identical, the device is in its "parallel" configuration, and offers only a small resistance to the flow of electrons with energies above the gap. The illustration in Fig. 1 shows the device in its "anti-parallel" configuration, which is realized when the polarity of the gates changes sign across the device. This produces a corresponding rotation of the pseudospin polarization with a switching of the out-of-plane component. Similarly to spin scattering at domain walls 21, the pseudospin of an incoming electron will precess about the changing local polarization as it attempts to follow it. If the change in the polarization rotation is sharp enough, the re-alignment of the electron's pseudospin should only be partially successful, leading to reflection and a drop in the flow of current through the device.

We characterize the fidelity of the pseudospin valve in terms of the pseudo-magnetoresistance (PMR) ratio

$$
\mathrm{PMR}=\frac{R_{\mathrm{AP}}-R_{\mathrm{P}}}{R_{\mathrm{AP}}},
$$

which is defined by the resistances $R_{\mathrm{P}}$ (parallel configuration) and $R_{\mathrm{AP}}$ (antiparallel configuration) determining the current $I=V / R$ flowing through the device in response to an applied bias voltage difference $V$. The PMR resistance ratio is the analogue of the conservative definition of magnetoresistance in spintronic applications [10, 21, and takes the value $100 \%$ for a perfect spin valve.

As in conventional spintronic applications, we also consider how the pseudospin-valve effect can be extended to a broader range of energies via serial connection of regions of different polarity. This leads to the design of a pseudospin-valve transistor operated by switching the polarity of a central gate of length $l$ (shown in Fig. 22).

Numerical results. - We start with numerical results, which are based on the microscopic tight-binding model of bilayer graphene. This model offers an accurate description of electronic transport in terms of a small number of characteristic energies and length scales. Transport between carbon atoms in a single layer (arranged

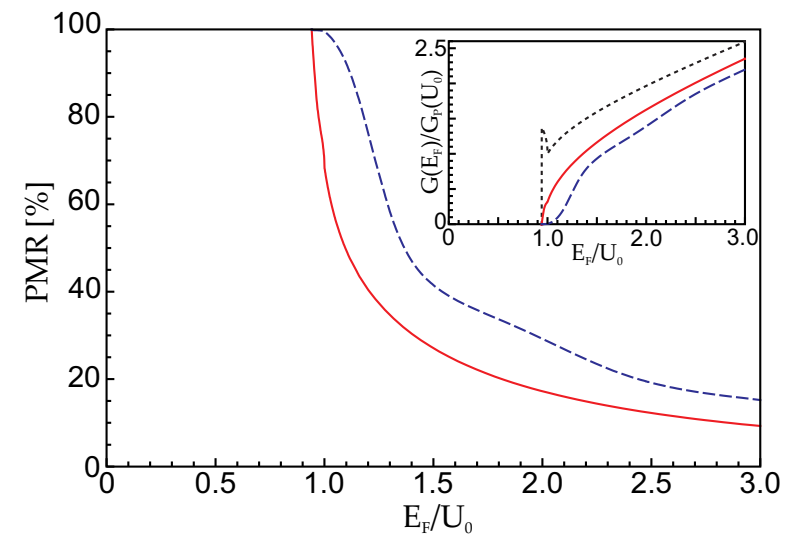

FIG. 3: Pseudo-magnetoresistance ratio $\mathrm{PMR}=\left(R_{\mathrm{AP}}-\right.$ $\left.R_{\mathrm{P}}\right) / R_{\mathrm{AP}}$ of the pseudospin valve (where $\mathrm{P}$ refers to the parallel configuration) versus the Fermi level of incoming electrons for $d=50 a$ (solid curve). Here, $U_{0}=0.07 \mathrm{eV}$ is the magnitude of the gate potential at large distances. The dashed curve refers to the pseudospin-valve transistor shown in Fig. 2 , with $l=50 a$. Inset: Conductance versus Fermi energy in the antiparallel (solid and dashed curves) and parallel (dotted curve) configurations from which the PMR is derived. The normalization factor is $G_{\mathrm{P}}\left(E_{F}=U_{0}\right)=4 U_{0} W /\left(3 \pi a \gamma_{0}\right)$.

on a honeycomb lattice with bond length $a=1.42 \AA$ ) is described by kinetic hopping energy $\gamma_{0} \approx 2.9 \mathrm{eV}$, which also determines the Fermi velocity $v_{0}=(3 / 2) a \gamma_{0} / \hbar$ of an isolated monolayer. In a bilayer, the two sheets of carbon are arranged according to Bernal stacking, whereby half of the atoms are strongly coupled to an atom in the other layer, with a strength determined by the interlayer coupling parameter $\gamma_{1} \approx 0.39 \mathrm{eV}$. Additional next-nearest neighbor couplings are non-essential for the problem at hand, and are therefore neglected for simplicity.

In the parallel configuration, the spatially constant onsite potential takes the value $U_{\text {top }}=U_{0}$ in the top layer and $U_{\text {bottom }}=-U_{0}$ in the bottom layer. In the antiparallel configuration of the device we model the on-site potential by

$$
U_{\text {top }}(x)=-U_{\text {bottom }}(x)=U(x) \equiv-U_{0} \operatorname{erf}(x / d),
$$

where erf is the error function and $x$ is the coordinate in the direction of transport. The main design parameters of the pseudospin valve are the typical length scale $d$ of variation of the gate potential and the magnitude $U_{0}$ of the potential value at large distance, as well as the Fermi energy $E_{F}$ which determines the energy at which the electrons are injected from the electrodes $\left(E_{F}=0\right.$ for a charge-neutral gapless bilayer). In the parallel configuration, the presence of a homogeneous symmetry-breaking onsite potential opens an energy gap $2\left|U_{0}\right| / \sqrt{1+\left(2 U_{0} / \gamma_{1}\right)^{2}}$ around the Fermi energy of the charge-neutral bilayer (see again Fig. 1). For the antiparallel configuration with the inhomogeneous potential $U(x)$ of Eq. (2), the solution of the tight-binding model requires, in general, a numerical approach. 
Since $U$ is $y$-independent, the problem of an infinitely wide ribbon is separable, and electronic modes with fixed transverse wavenumber $k_{y}$ decouple. For each transverse mode, the problem can be reduced to a one-dimensional chain of coupled bilayer unit cells, where each unit cell is composed of four carbon atoms. The Green function of each chain can be computed efficiently using the recursive Green function technique [22], which delivers the transmission amplitude $t\left(k_{y}\right)$ via the Fisher-Lee formula 23 . In the linear response regime, the total phase-coherent conductance of the nanoribbon is then obtained from the Landauer formula. For a ribbon of finite width $W$, the following considerations remain valid as long as $W \gg d$ and $k_{F} W \gg 1$, so that the contribution of edges can be neglected (see the discussion at the end of the paper).

The calculated conductance versus Fermi energy is shown in the inset of Fig. 3 for the parallel configuration, as well as the antiparallel configuration with one or two interfaces. In these calculations, the interface parameters are $d=50 a$ and $d=l=50 a$, respectively, and the asymptotic gap is $U_{0}=0.07 \mathrm{eV}$, corresponding to parameters which can be realized in present bilayer experiments [13, 19]. We find that close to the band edge the conductance in the antiparallel configuration is strongly reduced below its value in the parallel configuration. The resulting PMR ratio is plotted in the main panel of Fig. 3. For energies just above the gap, the PMR peaks at $100 \%$. For increasing Fermi energy the resistance ratio drops, which can be attributed to the decreasing out-ofplane component of the pseudospin of incoming electrons as they become less sensitive to the asymmetry of the layers when their kinetic energy increases. As expected from the GMR analogy, the presence of a second interface in the pseudospin-valve transistor (dashed line) significantly extends the energy range over which the PMR is $\approx 100 \%$.

Analytical considerations. - A qualitative analysis of the pseudospin-valve effect can be achieved by considering the low-energy physics of gapped graphene bilayers. The microscopic tight-binding Hamiltonian delivers a band structure with four bands. For realistic values of the charge carrier density, the Fermi surface of bilayer graphene lies in the vicinity of two valleys, indexed by $\xi= \pm 1$, situated at the $\mathrm{K}$ and $\mathrm{K}^{\prime}$ point at the corners of the hexagonal Brillouin zone. Owing to the interlayer coupling, two of the bands are split away by an energy $\approx \pm \gamma_{1}$. For the interlayer asymmetries and Fermi energies $\left|U_{0}\right|,\left|E_{F}\right| \ll \gamma_{1}$ assumed in this work, these split bands do not contribute to the electronic transport. In the absence of layer asymmetry, the two remaining bands touch at zero energy and have an approximately parabolic dispersion relation $E \approx \pm p^{2} / 2 m$, with effective mass $m=\gamma_{1} / 2 v_{0}^{2}$ and corresponding Fermi velocity $v_{F} \approx 2 v_{0} \sqrt{|E| /\left|\gamma_{1}\right|}$.

To explain the influence of interlayer asymmetry, we employ a two-component Hamiltonian [12] that approximately describes the electronic behavior in these two low-energy bands,

$$
H_{2} \approx-\frac{1}{2 m}\left(\begin{array}{cc}
0 & \left(\xi p_{x}-i p_{y}\right)^{2} \\
\left(\xi p_{x}+i p_{y}\right)^{2} & 0
\end{array}\right)+\left(\begin{array}{cc}
U & 0 \\
0 & -U
\end{array}\right) .
$$

The effective Hamiltonian $\mathrm{H}_{2}$ operates in a space of twocomponent wave functions $\Psi$ describing electronic amplitudes on the top and bottom layers. The first term in the Hamiltonian corresponds to a pseudospin-orbit coupling and ensures chirality of the electronic states in the absence of a symmetry-breaking on-site potential. The second term in $\mathrm{H}_{2}$ takes into account the influence of external gates that produce different on-site energies $\pm U$ on the two layers. This term is analogous to the Zeeman energy of a physical spin in a magnetic field parallel to the $z$ direction and leads to a gap $2|U|$ in the electronic spectrum $E_{ \pm} \approx \pm\left[U^{2}+\left(p^{2} / 2 m\right)^{2}\right]^{1 / 2}$. The pseudospin part of the corresponding wave functions takes the form

$$
\Psi_{ \pm}=\frac{1}{\sqrt{2}}\left(\begin{array}{c}
\sqrt{1+U / E} e^{-i \xi \phi} \\
\mp \sqrt{1-U / E} e^{i \xi \phi}
\end{array}\right)
$$

where $\phi$ is the angle of the momentum in the plane $\mathbf{p}=$ $(p \cos \phi, p \sin \phi)$. The pseudospin of such a state is

$$
\langle\boldsymbol{\sigma}\rangle=\mp \sqrt{1-\left(\frac{U}{E}\right)^{2}}(\hat{\imath} \cos 2 \phi+\hat{\jmath} \xi \sin 2 \phi)+\hat{k} \frac{U}{E} .
$$

For energy near the vicinity of the gap $|E| \approx|U|$, the outof-plane component takes its maximum value $\left\langle\sigma_{z}\right\rangle \approx 1$, whereas it is reduced away from the gapped region.

The pseudospin-valve effect proposed in the present paper originates in the large resistance at interfaces between regions of opposite preferred pseudospin direction. This resistance arises because the pseudospin degree of freedom can adjust itself to such a spatial variation only over a distance $l_{s}=h v_{0} / \sqrt{\left|E \gamma_{1}\right|}$, as follows from the scaling of the different terms in the two-component model. This pseudospin precession length scale is comparable to the Fermi wavelength, which is the scale on which chirality is established in the symmetric bilayer.

In both devices studied, the amount of reflection of incoming electrons in the anti-parallel configuration will therefore depend on the sharpness of the interface $d$ as compared to the pseudospin precession length $l_{s}$. Figure 4 shows the computed dependence of the resistance ratio on $d$ for fixed values of $E_{F}=U_{0}=0.07 \mathrm{eV}$. For these parameters, the pseudospin precession length $l_{s} \approx 165 \mathrm{a}$. In the regime $d / l_{s}<1$ of an abrupt interface, the electron's pseudospin is not able to rotate quickly enough to accommodate the change, which causes reflection and a large spin-valve effect. The series connection of two interfaces in the transistor further increases the resistance ratio to almost $100 \%$, except for very small values of $d$ where the resistance in the antiparallel arrangement drops due to tunneling through the central region. In the opposite 


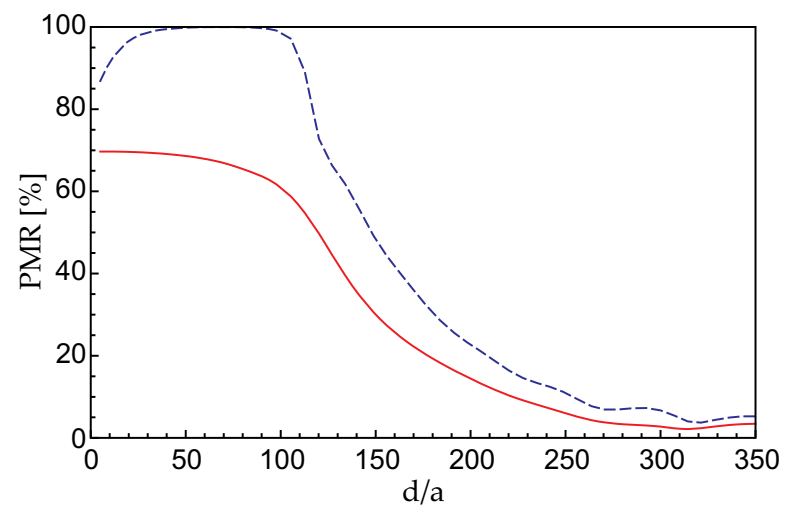

FIG. 4: Pseudo-magnetoresistance ratio of the pseudospin valve (solid curve) and the pseudospin-valve transistor (dashed curve) as a function of the sharpness $d$ of the interface between regions of different polarity. The Fermi energy is set to $E_{F}=U_{0}=0.07 \mathrm{eV}$.

limit $d / l_{s} \gg 1$, the pseudospin of incoming electrons is able to adiabatically adapt itself to the change of local polarization. In this limit the pseudospin-valve effect becomes negligible both for the single interface as well as for the series connection of two interfaces. The numerical results confirm that the transition between both regimes occurs at $d \approx l_{s}$. The pseudospin-valve effect can therefore be realized in devices with gate separation $d$ of the order of a few tens of nanometers.

Discussion and Conclusions. - The large mobility of charge carriers in graphene has stimulated intense research efforts that aim at the realization of graphenebased electronic devices. In particular, manipulation of the differential population of valley states in momentum space has been proposed [24], leading to a "valleytronic" analogy of spintronics. Our proposal of bilayer-based pseudospintronics relies on differential population of atomic orbitals in real space. This offers a robust mechanism to exploit spintronic analogies without the necessity of carefully fabricated nanoribbon edges, which limit the scalability of valleytronics 25] and induce harmful intervalley scattering [26].

In particular, pseudospintronics relies on bulk effects which do not depend on the crystallographic orientation of the interface. For the wide samples considered here $\left(W \gg \lambda_{F} \simeq l_{s} \gtrsim d\right)$, effects from the sample edges can be neglected since (i) edge states are localized at realistic rough edges, (ii) hypothetical clean edges at most contribute an additional transport channel per spin, and (iii) intervalley scattering off the edges can contribute to pseudospin relaxation across the interface, but this effect is negligible for $d \ll W$. Pseudospintronics is also remarkably robust against bulk disorder. Chirality guarantees that the bulk pseudospin-flip rate for majority carriers in a clean ballistic bilayer is zero (the out-of plane polarization of the pseudospin in the leads is valleyindependent). The predominant scattering mechanism in graphene, Coulomb scattering off charged impurities, does not break chirality [27, 28]. Inter-valley scattering contributes to pseudospin-flip scattering in the interface region, but the scattering lengths $l_{K K^{\prime}} \simeq 500 \mathrm{~nm}$ reported in recent experiments 29 indicates that this does not add an additional constraint on $d$.

Additional advantages of the proposed bilayer pseudospintronics concept arise from the fact that the charge carrier densities are finite. In devices that involve positioning the Fermi level within the bandgap, including field-effect transistors 18 or valley filters based on topologically-confined channels between insulating regions [30, the effective gap size is reduced by screening [16. 17]. Under these conditions, the largest gaps observed so far in experiment are of the order of $2 U_{0} \approx$ $10 \mathrm{meV} \approx 100 \mathrm{~K}$ [18. A finite charge density admits far larger gaps with experimental values reaching $2 U_{0} \approx 200 \mathrm{meV} \approx 2000 \mathrm{~K}[13$, 19] at high density.

We thank V. I. Fal'ko and C. Poole for helpful discussions. This research was supported by the European Commission, Marie Curie Excellence Grant MEXT-CT2005-023778, and by EPSRC First Grant EP/E063519/1.

[1] K. S. Novoselov et al., Science 306, 666 (2004).

[2] K. S. Novoselov et al., Nature 438, 197 (2005).

[3] Y. B. Zhang, Y. W. Tan, H. L. Stormer and P. Kim, Nature 438, 201 (2005).

[4] A. K. Geim and K. S. Novoselov, Nature Mater. 6, 183 (2007).

[5] K. S. Novoselov et al., Nature Phys. 2, 177 (2006).

[6] T. Ando, T. Nakanishi, and R. Saito, J. Phys. Soc. Jpn. 67, 2857 (1998).

[7] P. L. McEuen et al., Phys. Rev. Lett. 83, 5098 (1999).

[8] M. I. Katsnelson, K. S. Novoselov, and A. K. Geim, Nature Phys. 2, 620 (2006).

[9] V. V. Cheianov and V. I. Fal'ko, Phys. Rev. B 74, 041403(R) (2006).

[10] M. A. M. Gijs and G. E. W. Bauer, Advances in Physics 46, 285 (1997).

[11] D. Loss and D. P. DiVincenzo, Phys. Rev. A 57, 120 (1998).

[12] E. McCann and V. I. Fal'ko, Phys. Rev. Lett. 96, 086805 (2006).

[13] T. Ohta et al., Science 313, 951 (2006).

[14] H. Min, G. Borghi, M. Polini and A. H. MacDonald, Phys. Rev. B 77, 041407(R) (2008).

[15] F. Guinea, A. H. Castro Neto, and N. M. R. Peres, Phys. Rev. B 73, 245426 (2006).

[16] E. McCann, Phys. Rev. B 74, 161403(R) (2006).

[17] H. Min, B. Sahu, S. K. Banerjee and A. H. MacDonald, Phys. Rev. B 75, 155115 (2007).

[18] J. B Oostinga, H. Heersche, X. Liu and A. Morpurgo, Nature Mater. 7, 151 (2007).

[19] E. V. Castro et al., Phys. Rev. Lett. 99, 216802 (2007).

[20] M. N. Baibich et al., Phys. Rev. B39, 4828 (1989).

[21] M. Viret et al., Phys Rev. B 53, 8464 (1996).

[22] S. Datta, Electronic Transport in Mesoscopic Systems 
(Cambridge University Press, Cambridge, 1997).

[23] D. S. Fisher, P. A. Lee, Phys. Rev. B 23, 6851 (1981).

[24] A. Rycerz, J. Tworzydlo, and C. W. J. Beenakker, Nature Phys. 3, 172 (2007).

[25] V. I. Fal'ko, Nature Phys. 3, 151 (2007).

[26] A. R. Akhmerov, J. H. Bardarson, A. Rycerz, and C. W. J. Beenakker, Phys. Rev. B 77, 205416 (2008).

[27] S. V. Morozov et al., Phys. Rev. Lett. 100, 016602 (2008).

[28] S. Adam and S. Das Sarma, Phys. Rev. B 77, 115436 (2008).

[29] R. V. Gorbachev et al., Phys. Rev. Lett. 98, 176805 (2007).

[30] I. Martin, Ya. M. Blanter, and A. F. Morpurgo, Phys. Rev. Lett. 100, 036804 (2008). 